\title{
Volume of Distribution of Fraction Dose Predicted Normalized by Dose
}

National Cancer Institute

\section{Source}

National Cancer Institute. Volume of Distribution of Fraction Dose Predicted Normalized

by Dose. NCl Thesaurus. Code C102730.

The volume of distribution associated with the terminal slope following extravascular administration divided by the fraction of dose absorbed, calculated using the predicted value of the last non-zero concentration, divided by the dose. 